\title{
Tiempos y rendimiento en el proceso de aserrado de Eucalyptus globulus Labill, con sierra circular y de cinta
}

\author{
(Time and yield on the sawing process \\ of Eucalyptus globulus Labill, with circular and band saw)
}

\author{
Miguel Guallpa' ${ }^{1}$ José Suatunce ${ }^{2}$, Hayron Canchignia²
}

\begin{abstract}
Resumen
Con el objetivo de determinar los tiempos y rendimiento en el proceso de transformación de madera en rollo a madera escuadrada de Eucalyptus globulus se realizó una investigación utilizando sierra circular (aserradero A) y sierra circular más sierra de cinta (aserradero B). Los tamaños de las muestras fueron de 210 y 205 trozas. Para conocer el tiempo de conversión de las trozas se utilizó el método de vuelta a cero y en el rendimiento se relacionó el volumen aserrado con el volumen en rollo de las trozas. Los resultados indican un comportamiento significativo, cuyos tiempos al procesar $1.000 \mathrm{pt}\left(2.36 \mathrm{~m}^{3}\right)$ son de 112.73 con sierra circular y 167.36 minutos mediante el sistema sierra circular más la sierra de cinta. Con rendimientos de $27.74 \%$ en el aserradero A y de $48.84 \%$ para el aserradero B, es decir, por cada metro cúbico de madera en rollo $\left(\mathrm{m}^{3}\right.$. r) procesada, se obtuvo 117 pt y 207 pt de madera aserrada, respectivamente. Finalmente, en el aserradero A, a medida que aumentó el diámetro de las trozas, se incrementó el rendimiento y productividad, mientras que en el aserradero B, disminuyó el rendimiento y mostró un comportamiento variable la productividad, debido al diferente número de trozas evaluadas por clase diamétrica en cada fábrica.
\end{abstract}

\section{Palabras clave}

Aserraderos; Eucalyptus globulus; madera aserrada; tipo de sierra

\begin{abstract}
In order to determine the times and performance in the transformation process of round wood to squared wood of Eucalyptus globulus, a research was carried using circular saw (sawmill A) and circular saw more band saw (sawmill B). The sizes of the samples were 210 and 205 logs. To know the conversion time of the logs, the return-to-zero method was used and in the yield the sawn volume was related to the roll volume of the logs. The result indicate a significant behavior, whose times when processing 1.000 pt $(2.36 \mathrm{~m}$ 3) are of 112.73 with circular saw and 167.36 minutes by means of the circular saw system plus the band saw. With yields of $27.74 \%$ in the sawmill $A$ and $48.84 \%$ in the sawmill B, means that for each cubic meter of round wood (m3. r) processed, 117 pt and 207 st of sawn wood were obtained respectively. Finally, in the sawmill $A$, as the diameter of the logs increases, the yield and productivity increase. However, in the sawmill B, the yield diminishes and the productivity showed a variable behavior, due to the different number of logs evaluated by diameter class in each factory.
\end{abstract}

\section{Keywords}

Sawmills; Eucalyptus globulus; sawn timber; saw type

\section{Introducción}

En Ecuador, una de las especies mayormente utilizadas para la elaboración de madera aserrada es el Eucalyptus globulus proveniente de plantaciones forestales (López y Muñoz, 2017). En 2011 la producción de madera aserrada fue de $519000 \mathrm{~m}^{3}$, siendo el producto de mayor crecimiento con el 11 \% en comparación con 2010, seguido de madera en rollo con el $1 \%$ (PRO ECUADOR, 2013). Especialmente, se usa para la fabricación de muebles e insumos para la construcción (Ministerio del Ambiente, 2011). 
Se obtiene madera dimensionada al utilizar aserraderos de cinta o de disco. En cada fábrica de aserrío es necesario combinar recursos como la maquinaria, recurso humano, fuentes de energía y dinero; durante un tiempo y derivado de un diferente nivel tecnológic son los factores que influyen en la productividad de la transformación primaria y que inciden en los costos de producción (García et al., 2001)

Al estudiar la productividad Pinus taeda, con los sistemas de aserradero convencional y optimizado, obtuvieron rendimientos de $44.21 \%$ y 53.60 \% respectivamente (Murara et al., 2005).

En Riobamba, no se dispone de datos oficiales sobre porcentaje de aprovechamiento por $\mathrm{m}^{3}$ de madera en rollo al utilizar sierras circulares y de cinta o su combinación. Los reportes de investigaciones en eucalipto corresponden a evaluaciones cuantitativas y cualitativas de masas forestales (Guallpa et al., 2016, 2019). Otro estudio indica el nivel de consumo de residuos de eucalipto en la región interandina con valores entre el 34-41.03 \% (Guallpa et al., 2018). De acuerdo a lo expuesto, la industria del aserrío en Riobamba requiere información técnica para definir estrategias de manejo y aprovechamiento de trozas de madera de la especie forestal en estudio.

Siendo el tipo de aserrío utilizado, el que determina el grado de aprovechamiento de la materia prima (Ecuador forestal, 2013); este rendimiento en madera aserrada es la relación entre el volumen de madera aserrada obtenido y el volumen de la troza antes del aserrío (Valerio et al., 2007).

Su eficiencia está ligada al tipo de sierra que se emplea más otros factores como el nivel tecnológico de las máquinas utilizadas, el tipo de aserradero, el tipo de procesado de especies (coníferas o latifoliadas) y el diámetro de las trozas pueden afectar el rendimiento de madera obtenida a nivel de aserradero (Vital, 2008).

Aunque la fábrica que no se ocupe de mejorar sus rendimientos y consecuentemente reducir sus costos de producción, asume un serio riesgo de perder en competitividad y paralizar sus actividades por ineficiente (Biasi y Pereira da Rocha, 2007).

La tecnología utilizada incide en el grado de generación y acumulación de residuos forestales propiciando impactos negativos en el aspecto económico y ambiental de las fábricas. En tal sentido, el conocimiento de los indicadores de productividad es de interés en toda administración ya que posibilita que las decisiones sobre el desempeño industrial y uso eficiente de la materia prima sean tomadas anticipadamente ante situaciones adversas, reduciendo gastos y pérdidas en el proceso productivo (Valério et al., 2009).

En la ciudad de Riobamba, con base en la información proporcionada por la oficina Técnica del Ministerio del Ambiente Chimborazo (2018) y verificación in situ funcionan alrededor de 30 aserraderos, de los cuales 18 aserraderos (60 \%) cuentan con una sierra principal industrial; 4 con sierra circular (13.33 \%) y los 8 restantes (26.67 \%) están provistos de una sierra principal y de cinta para la transformación de madera rolliza a madera aserrada; sin embargo, no se conoce el efecto que tienen el diámetro, la longitud y la conicidad de la trocería en el grado de aprovechamiento de la materia prima entrante versus las tablas que se obtienen frente a la cantidad de residuos que se generan a causa de la tecnología empleada en el proceso de transformación primaria de la madera, los impactos económicos y ambientales como consecuencia del tipo de tecnología, son las razones que motivaron evaluar los tiempos y rendimientos del proceso de aserrío de madera de eucalipto, y a la vez valorar los tiempos, rendimientos por diámetro y conicidad de las trozas con el propósito de plantear directrices que permitan a mediano y largo plazo incrementar el nivel de productividad. 


\section{Materiales y métodos}

Por las características de las instalaciones con las que cuentan doce aserraderos, se agruparon en cuatro con sierra circular y ocho restantes disponen de una sierra circular y de cinta constituyeron la población objeto de estudio, se consideró la selección de dos aserraderos, el primero se denomina A, está provisto de una sierra circular y el segundo se le nombra B, el cual posee una sierra circular y otra de cinta.

Los dos aserraderos son de propiedad particular y están dedicados a la elaboración y venta de madera aserrada de E. globulus, mayormente tablas. Físicamente, las instalaciones se sitúan dentro del cantón Riobamba. El aserradero A se encuentra ubicado a $5 \mathrm{~km}$ de la vía Riobamba-Quimiag cerca al desvío a San Gerardo y el aserradero B se localiza a dos cuadras del mercado mayorista de la ciudad de Riobamba.

Geográficamente se ubican en los puntos de referencia: UTM Zona 17S Datum WGS 84 (Tabla 1).

Tabla 1. Ubicación geográfica de los aserraderos del estudio

\begin{tabular}{|c|c|c|c|}
\hline Aserraderos & X & Y & Altitud (msnm) \\
\hline A & 764353 & 9817384 & 2750 \\
\hline B & 763654 & 9813400 & 2745 \\
\hline
\end{tabular}

En la investigación se aplicaron los métodos: documental, exploratoria, descriptiva y de campo (Calvache, 2016).

El muestreo de las variables cuantitativas tuvo lugar el primer semestre del año 2018.

\section{1. Determinación del tamaño de muestra}

En el presente estudio se llevó a cabo un premuestreo con 50 trozas en cada aserradero, con lo cual fue posible estimar el número de trozas necesarias por aserradero (Tabla 2), para alcanzar un error de muestreo menor al $10 \%$ y una confiabilidad de $90 \%$, se utilizó la Ecuación 1 (Rondeux, 2010).

$$
\mathbf{n}=\frac{\mathrm{t}^{2} C V^{2}}{E^{2}}
$$

Donde:

$\mathrm{n}$ = Número de trozas necesarias para estimar el rendimiento de madera aserrada

$\mathrm{t}=$ Valor tabular de $\mathrm{t}$-Student a $90 \%$ de confiabilidad

$\mathrm{CV}=$ Coeficiente de variación (\%)

$\mathrm{E}=$ Error de muestreo deseado $(\%)$

Tabla 2. Número de trozas requeridas por aserradero evaluado

\begin{tabular}{|l|c|c|c|}
\hline \multicolumn{1}{|c|}{ Aserradero } & Trozas requeridas $(\mathbf{n})$ & Coeficiente de variación (\%) & Error de muestreo (\%) \\
\hline A & 210 & 23.03 & 2.62 \\
\hline B & 205 & 32.98 & 3.81 \\
\hline Suma & 415 & & \\
\hline
\end{tabular}




\subsection{Selección y marcado de trozas}

La selección de las trozas se realizó al azar. Luego de elegidas las trozas a ingresar a la fase de corte se marcaron sus extremos con tiza mediante un número asignado, 15 minutos antes que se inicie con el aserrío de las muestras (Figura 1).

Figura 1. Códigos para la identificación de las muestras

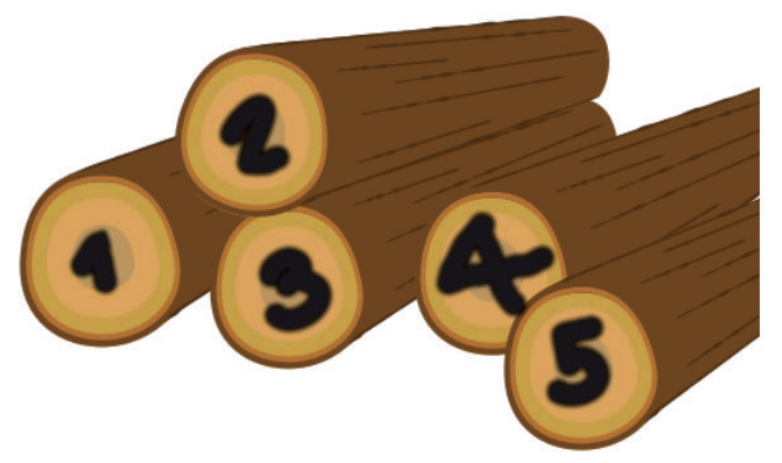

Fuente: (INAB, ITTO, 2016)

\subsection{Medición de las trozas}

Se realizó con una cinta diamétrica y un flexómetro en el caso de la longitud (Figura 2).

Figura 2. Medición de una sección de madera en rollo

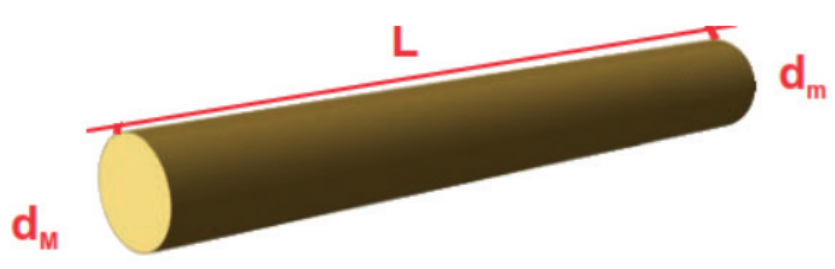

Donde:

$\mathrm{dM}=$ Diámetro mayor

$\mathrm{dm}=$ Diámetro menor

$L=$ Longitud

\subsection{Medición de volumen de madera}

Mediante la toma de medidas del espesor, ancho, longitud del producto resultante y la cuantificación del número de tablas obtenidas por troza. 
Figura 3. Medición de una pieza de madera aserrada

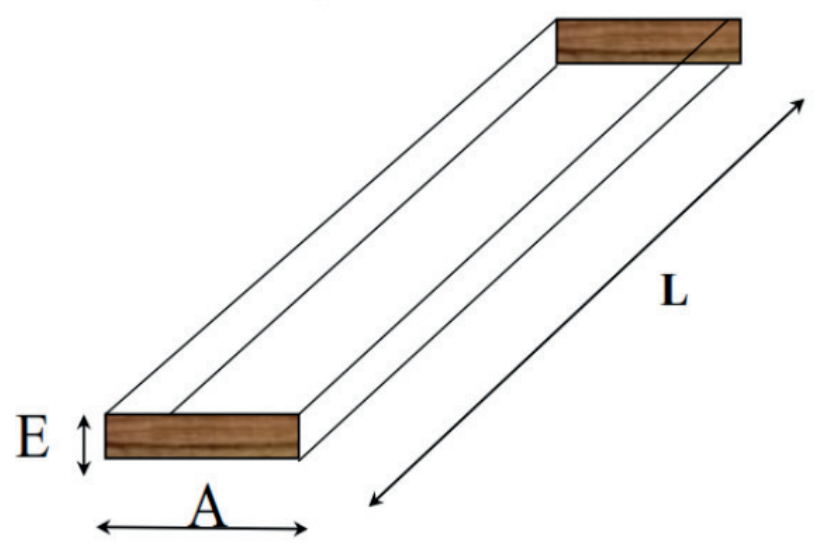

Fuente: (Ecuador forestal, 2010)

Donde:

$E=$ Espesor

$A=$ Ancho

$\mathrm{L}=$ Largo

\subsection{Registro de tiempos y movimientos}

Se utilizó el método de "vuelta a cero", descrito por Villagómez y García (1986), el cual corresponde a los métodos de muestreo aleatorio, luego de conocer el esquema de operación que se aplica para el proceso de transformación de la madera en cada aserradero se registró los siguientes tiempos y movimientos (Tabla 3).

Tabla 3. Tiempos y movimientos identificados y registrados en los dos aserraderos

\begin{tabular}{|l|l|l|}
\hline \multicolumn{1}{|c|}{ Aserradero A } & \multicolumn{1}{|c|}{ Aserradero B } & \multicolumn{1}{c|}{ Aserradero B } \\
\hline Proceso completo & Primera fase: sierra circular & Segunda fase: sierra de cinta \\
\hline $\begin{array}{l}\text { Tiempo de descargue de trozas } \\
\text { en aserradero }\end{array}$ & $\begin{array}{l}\text { Tiempo de descargue de trozas en } \\
\text { aserradero }\end{array}$ & Tiempo de carga a sierra de cinta \\
\hline $\begin{array}{l}\text { Tiempo de traslado de troza a la } \\
\text { sierra circular }\end{array}$ & $\begin{array}{l}\text { Tiempo de traslado de troza a la sie- } \\
\text { rra circular }\end{array}$ & Tiempo de avance \\
\hline Tiempo de carga & Tiempo de carga & Tiempo de retroceso \\
\hline Tiempo de avance & Tiempo de avance & $\begin{array}{l}\text { Tiempo de volteos para colocar bloques } \\
\text { en la parte superior de la sierra de cinta }\end{array}$ \\
\hline Tiempo de retrocesos & Tiempo de retrocesos & $\begin{array}{l}\text { Tiempo de traslado y apilado de tablas } \\
\text { que ingresaron a la sierra de cinta }\end{array}$ \\
\hline Tiempo de volteos de las trozas & Tiempo de volteos de las trozas & Tiempo justificado en la sierra de cinta \\
\hline $\begin{array}{l}\text { Tiempo de traslado y apilado de } \\
\text { tablas }\end{array}$ & $\begin{array}{l}\text { Tiempo de traslado y apilado de } \\
\text { bloques }\end{array}$ & $\begin{array}{l}\text { Tiempo no justificado en la sierra de } \\
\text { cinta }\end{array}$ \\
\hline Tiempo justificado & Tiempo Justificado & \\
\hline Tiempo no justificado & Tiempo no justificado & \\
\hline
\end{tabular}


A continuación, se describen los tiempos y movimientos registrados en la investigación.

\subsection{Tiempo productivo}

\section{Tiempo de descargue}

Tiempo en segundos que se demora en colocar la troza desde el camión hasta el sitio de almacenamiento provisional.

\section{Tiempo de traslado de troza a equipo}

Se registró al tiempo que tomó llevar la troza desde el área de almacenamiento provisional hasta el punto donde se carga la troza al carrete, corresponde una distancia de $2 \mathrm{~m}$ aproximadamente.

\section{Tiempo de carga}

Se cronometró el tiempo que tomó subir la troza al carrete, la colocación del primer gancho maderero en la troza para acercarla y asegurarla al carro escuadra.

\section{Tiempo de avance del carro escuadra}

Se contabilizó el tiempo en que la troza inició el movimiento hacia la sierra circular y culminó cuando se detuvo el movimiento del carro escuadra. Este puede variar en 3 o más tiempos dependiendo de las dimensiones de la troza.

\section{Tiempo de retroceso del carro escuadra}

Se registró a partir del regreso del carro escuadra a la posición inicial para empezar un nuevo avance. Las veces que el carro escuadra regrese a la posición inicial.

Tiempo de volteos de las trozas en el carro escuadra

Se tomó en el momento de contacto del gancho maderero para girar la troza en otra posición para buscar otros planos de corte. Este tiempo vario de tres tiempos en adelante dependiendo de las dimensiones de la troza.

\section{Tiempo justificado}

Corresponde el tiempo invertido en mantenimiento u otra actividad para resolver imprevistos propios de trabajo.

\subsection{Tiempo improductivo}

\section{Tiempo no justificado}

Se consideró el tiempo empleado para realizar actividades ajenas al proceso de aserrío como descansos, conversación entre operarios y otras distracciones no productivas durante el proceso.

Para la segunda fase de la sierra de cinta se aplicó criterios similares en el registro de los tiempos requeridos de cada sub actividad durante el proceso de conversión de madera en trozas a tablas. 


\subsection{Cubicación de las trozas}

A partir de los datos de sus diámetros: mayor, menor y su longitud, se determinó su volumen individual con corteza, empleando la fórmula de Smalian (Rivas, 2013), mediante la Ecuación 2:

$$
\mathrm{V}=\frac{\left(\pi / 4 \times \mathrm{DM}^{2}\right)+\left(\pi / 4 \times \mathrm{Dm}^{2}\right)}{2} \times \mathrm{L}
$$

Donde:

$$
\begin{aligned}
& \mathrm{V}=\text { Volumen de la troza }\left(\mathrm{m}^{3}\right) \\
& \mathrm{DM}=\text { Diámetro mayor de la troza }(\mathrm{m}) \\
& \mathrm{Dm}=\text { Diámetro menor de la troza }(\mathrm{m}) \\
& \mathrm{L}=\text { Longitud de la troza }(\mathrm{m}) \\
& \mathrm{pi}=\text { Constante }(3.14159)
\end{aligned}
$$

\subsection{Determinación del tiempo de aserrado de 1,000 pies tablares $\left(2.36 \mathrm{~m}^{3}\right)$}

A partir de la información generada en el aserrado de las trozas se determinó el tiempo necesario para aserrar 1.000 pt (2.36 m³) con la Ecuación 3 (Nájera et al., 2011a): 1 $\mathrm{m}^{3}$ de madera = 424 pt.

$$
T=\frac{1000 T t}{V a}
$$

Donde:

$\mathrm{T}=$ Tiempo para aserrar 1.000 pies tablares (min.)

$\mathrm{Tt}=$ Tiempo total de aserrado (min.)

$\mathrm{Va}=$ Volumen aserrado $(\mathrm{pt})$

\subsection{Cubicación de las piezas aserradas}

Basándose en las medidas registradas de las piezas resultantes del aserrío se calculó el volumen aserrado (Romahn et al., 1987, citado por Nájera et al., 2012) mediante la Ecuación 4:

\section{$\mathrm{Va}=(\mathrm{g})(\mathrm{a})(\mathrm{l})$}

Donde:

$$
\begin{aligned}
& \text { Va = Volumen de la pieza aserrada }\left(\mathrm{m}^{3}\right) \\
& \mathrm{g}=\text { Grueso de la pieza aserrada }(\mathrm{m}) \\
& \mathrm{a}=\text { Ancho de la pieza aserrada }(\mathrm{m}) \\
& \mathrm{l}=\text { Longitud de la pieza aserrada }(\mathrm{m})
\end{aligned}
$$

\subsection{Determinación del rendimiento de madera aserrada}

Con el volumen calculado a partir de las piezas aserradas y el volumen de la materia prima que se empleó, se determinó el rendimiento utilizando la Ecuación 5 (Quirós et al., 2005, citado por Nájera et al., 2012). 


$$
R=\frac{V t a}{V t r} 100
$$

\subsection{Categorías de diámetro y conicidad de las trozas}

A fin de establecer las categorías diamétricas de las trozas se consideraron todos los diámetros mínimos con corteza en los patios de almacenamiento provisional, encontrándose valores de 14 a $48 \mathrm{~cm}$, para lo cual se formaron cuatro categorías diamétricas con un rango de $10 \mathrm{~cm}$, y para la conicidad se establecieron seis categorías con intervalos de $1 \mathrm{~cm} / \mathrm{m}$. Además, se identificó que no se realiza una selección por diámetros de las trozas antes de iniciar con el proceso de aserrío (Tabla 4).

Tabla 4. Distribución de las trozas en categorías diamétricas y por conicidad

\begin{tabular}{|l|c|c|c|c|c|}
\hline $\begin{array}{c}\text { Categoría } \\
\text { diamétrica (cm) }\end{array}$ & \multicolumn{2}{|c|}{$\begin{array}{c}\text { Trozas } \\
\text { por categoría }(\mathbf{n})\end{array}$} & $\begin{array}{c}\text { Categoría } \\
\text { de conicidad }(\mathbf{c m} / \mathbf{m})\end{array}$ & \multicolumn{2}{c|}{$\begin{array}{c}\text { Trozas } \\
\text { por categoría }(\mathbf{n})\end{array}$} \\
\hline Aserradero & $\mathrm{A}$ & $\mathrm{B}$ & Aserradero & $\mathrm{A}$ & B \\
\hline $14-25$ & 75 & 129 & $0-1$ & 119 & 103 \\
\hline $25.1-35$ & 79 & 60 & $1.1-2$ & 84 & 85 \\
\hline $35.1-45$ & 56 & 14 & $2.1-3$ & 7 & 10 \\
\hline $45.1-55$ & & 2 & $3.1-4$ & & 4 \\
\hline & & & $4.1-5$ & & 2 \\
\hline & & & $5.1-6$ & & 1 \\
\hline Total & 210 & 205 & & 210 & 205 \\
\hline
\end{tabular}

\section{Resultados y discusión}

\subsection{Tiempos y rendimientos del proceso de aserrío de madera de eucalipto}

Los resultados del tiempo estimado para elaborar 1.000 pt oscilan desde 63.02 a $202.5 \mathrm{mi}$ nutos al trabajar con sierra circular en el aserradero A. En promedio se requiere $112.73 \mathrm{mi}-$ nutos de los cuales 79.78 corresponden al tiempo productivo, específicamente los avances, y retrocesos del carro escuadra para efectuar los cortes en las trozas más la actividad de volteo de las trozas al utilizar la sierra circular en el aserradero. En el aserradero A de las 210 trozas de eucalipto evaluadas a partir de un volumen de $39.03 \mathrm{~m}^{3} \mathrm{r}$ con corteza, se generaron 1254 tablas dando un volumen aserrado de $11.51 \mathrm{~m}^{3}$, valor que corresponde a $4875 \mathrm{pt}$ (Tabla 5). 
Tabla 5. Estimadores estadísticos de los tiempos y rendimientos con sierra circular del aserradero A

\begin{tabular}{|c|c|c|c|c|c|}
\hline Variables & Media & Desv Std & Máximo & Mínimo & Total \\
\hline \multicolumn{6}{|c|}{ Características de las trozas aserradas } \\
\hline Diámetro mayor con corteza (m) & 0.31 & 0.07 & 0.45 & 0.18 & \\
\hline Diámetro menor con corteza (m) & 0.28 & 0.07 & 0.42 & 0.17 & \\
\hline Longitud de la troza (m) & 2.52 & 0.03 & 2.57 & 2.48 & \\
\hline Volumen con corteza $\left(\mathrm{m}^{3} \cdot r\right)$ & 0.19 & 0.09 & 0.37 & 0.06 & 39.03 \\
\hline \multicolumn{6}{|l|}{ Productos obtenidos del aserrío } \\
\hline Tablas generadas ( $\mathrm{n}$ ) & 6 & 4 & 15 & 1 & 1254 \\
\hline Volumen de las tablas $\left(\mathrm{m}^{3}\right)$ & 0.05 & 0.03 & 0.15 & 0.01 & 11.51 \\
\hline Volumen aserrado (pt) & 23.22 & 14.56 & 62.43 & 3.64 & 4875 \\
\hline \multicolumn{6}{|c|}{ Tiempo para aserrar 1000 pt (min) en la sierra circular } \\
\hline $\begin{array}{l}\text { Tiempo de descargue de trozas en } \\
\text { aserradero }\end{array}$ & 13.74 & 6.800 & 30.58 & 2.67 & \\
\hline $\begin{array}{l}\text { Tiempo de traslado de troza a la sierra } \\
\text { circular }\end{array}$ & 2.52 & 0.390 & 5.35 & 2.30 & \\
\hline Tiempo de carga & 2.95 & 0.640 & 8.89 & 1.78 & \\
\hline Tiempo de avance & 49.56 & 19.020 & 84.78 & 24.60 & \\
\hline Tiempo de retrocesos & 15.44 & 8.160 & 26.86 & 2.30 & \\
\hline Tiempo de volteos de las trozas & 14.78 & 8.460 & 32.92 & 2.72 & \\
\hline Tiempo de traslado y apilado de tablas & 1.67 & 0.370 & 2.86 & 1.13 & \\
\hline Tiempo justificado & 9.88 & 6.490 & 40.20 & 0.83 & \\
\hline Tiempo no justificado & 2.19 & 0.920 & 5.31 & 0.86 & \\
\hline Tiempo total de aserrado & 112.73 & & & & \\
\hline $\begin{array}{l}\text { Tiempo total de aserrado para } 1000 \mathrm{pt} \\
\text { (min) }\end{array}$ & 112.73 & 34.16 & 202.5 & 63.02 & \\
\hline Rendimiento con corteza (\%) & 27.74 & 6.39 & 44.09 & 11.12 & \\
\hline Productividad $\left(\mathrm{m}^{3} \cdot \mathrm{h}^{-1}\right)$ & 1.17 & 0.47 & 2.57 & 0.25 & \\
\hline
\end{tabular}

En cambio, en el sistema sierra circular + sierra de cinta, requiere de 98.2 a 640.48 minutos en el aserradero B. Iniciando con la conversión de trozas a bloques en la sierra circular y posteriormente a tablas en la sierra de cinta, se estimó un tiempo total medio de 167.36 minutos, el tiempo productivo es de 46.16 minutos en la elaboración de bloques, mientras que en la fase complementaria de bloques a tablas su valor proyectado es de 73.07 minutos en la sierra de cinta. En el aserradero B de las 205 trozas de eucalipto evaluadas a partir de un volumen de $27.81 \mathrm{~m}^{3} \mathrm{r}$ con corteza, se generaron 1130 tablas, lo cual representa un volumen aserrado de $11.97 \mathrm{~m}^{3}$, valor que corresponde a 5069.6 pt (Tabla 6). 
Tabla 6. Estimadores estadísticos de los tiempos y rendimientos con sierra circular + sierra de cinta del aserradero B

\begin{tabular}{|c|c|c|c|c|c|}
\hline Variable & Media & $\begin{array}{c}\text { Desv } \\
\text { Std }\end{array}$ & Máximo & Mínimo & Total \\
\hline \multicolumn{5}{|c|}{ Características de las trozas aserradas } & \\
\hline Diámetro mayor con corteza (m) & 0.27 & 0.07 & 0.51 & 0.15 & \\
\hline Diámetro menor con corteza (m) & 0.24 & 0.06 & 0.47 & 0.14 & \\
\hline Longitud de la troza (m) & 2.46 & 0.12 & 2.56 & 2.15 & \\
\hline Volumen con corteza $\left(\mathrm{m}^{3} \cdot r\right)$ & 0.14 & 0.08 & 0.47 & 0.04 & 27.81 \\
\hline \multicolumn{6}{|l|}{ Productos obtenidos del aserrío } \\
\hline Tablas generadas ( $\mathrm{n}$ ) & 6 & 2 & 10 & 3 & 1130 \\
\hline Volumen de las tablas $\left(\mathrm{m}^{3}\right)$ & 0.06 & 0.02 & 0.17 & 0.02 & 11.97 \\
\hline Volumen aserrado (pt) & 24.73 & 8.85 & 73.14 & 10.43 & 5069.6 \\
\hline \multicolumn{5}{|c|}{ Tiempo para aserrar 1000 pt (min.) en la fase de sierra circular } & \\
\hline Tiempo de descargue de trozas en aserradero & 11.48 & 3.78 & 23.53 & 5.51 & \\
\hline Tiempo de traslado de troza a la sierra circular & 3.61 & 2.99 & 23.58 & 0.81 & \\
\hline Tiempo de carga & 4.32 & 3.74 & 25.04 & 1.42 & \\
\hline Tiempo de avance & 20.66 & 9.84 & 62.88 & 4.16 & \\
\hline Tiempo de retrocesos & 12.20 & 8.08 & 53.96 & 2.10 & \\
\hline Tiempo de volteos de las trozas & 13.30 & 35.77 & 513.64 & 1.42 & \\
\hline Tiempo de traslado y apilado de bloques & 1.92 & 0.28 & 2.76 & 1,21 & \\
\hline Tiempo Justificado & 15.47 & 6.24 & 43.81 & 6.84 & \\
\hline Tiempo no justificado & 4.36 & 0.95 & 7.47 & 3.02 & \\
\hline Subtotal proceso sierra circular & 87.32 & & & & \\
\hline \multicolumn{5}{|c|}{ Tiempo para aserrar 1000 pt (min.) en la fase de sierra de cinta } & \\
\hline Tiempo de carga a sierra de cinta & 2.52 & 1.31 & 5.93 & 0.91 & \\
\hline Tiempo de avance & 30.36 & 12.22 & 61.2 & 14,96 & \\
\hline Tiempo de retroceso & 28.93 & 12.89 & 61.2 & 11.08 & \\
\hline $\begin{array}{l}\text { Tiempo de volteos para colocar bloques en la parte } \\
\text { superior de la sierra de cinta }\end{array}$ & 13.78 & 6.42 & 30.91 & 6.98 & \\
\hline $\begin{array}{l}\text { Tiempo de traslado y apilado de tablas que ingresa- } \\
\text { ron a la sierra de cinta }\end{array}$ & 1.59 & 0.34 & 2.7 & 1.08 & \\
\hline Tiempo justificado en la sierra de cinta & 1.55 & 0.03 & 1.68 & 1.48 & \\
\hline Tiempo no justificado en la sierra de cinta & 1.32 & 0.04 & 1.46 & 1.01 & \\
\hline Subtotal proceso en sierra de cinta & 80.05 & & & & \\
\hline Total procesos de sierra circular y de cinta & 167.37 & & & & \\
\hline Tiempo total de aserrado para 1000 pt (min.) & 167.37 & 56.15 & 640.48 & 98.2 & \\
\hline Rendimiento con corteza (\%) & 48.84 & 16.11 & 121.8 & 10.11 & \\
\hline Productividad $\left(\mathrm{m}^{3} \cdot \mathrm{h}-{ }^{-1}\right)$ & 0.89 & 0.32 & 2.59 & 0.2 & \\
\hline
\end{tabular}


Las diferencias significativas de las variables: tiempo total de aserrado para $1000 \mathrm{pt}$ (min.), el rendimiento con corteza y la productividad entre los dos tipos de proceso de aserrío evaluados obtuvieron un valor $\mathrm{p}<0.0001$ (Tabla 7). Siendo mejor el tiempo total de aserrado el estimado con la sierra circular, su valor promedio es de 112,75 minutos.

Tabla 7. Prueba de Mann-Whitney a los indicadores de productividad en la operación de aserrío

\begin{tabular}{|c|c|c|c|c|c|c|c|}
\hline Variable & Aserradero & $N$ & Media & Desv Std & Mediana & W & $P$ \\
\hline \multirow{2}{*}{$\begin{array}{l}\text { Tiempo total de aserrado para } \\
1000 \text { pt }\end{array}$} & $A$ & 210 & 112.75 & 34.16 & 98.83 & \multirow{2}{*}{56414} & \multirow{2}{*}{0.0001} \\
\hline & $B$ & 205 & 167.36 & 56.15 & 149.59 & & \\
\hline \multirow{2}{*}{ Rendimiento con corteza } & $A$ & 210 & 27.74 & 6.39 & 27.95 & \multirow{2}{*}{59371} & \multirow{2}{*}{0.0001} \\
\hline & B & 205 & 48.84 & 16.11 & 49.94 & & \\
\hline \multirow{2}{*}{ Productividad del aserrío } & A & 210 & 1.17 & 0.47 & 1.16 & \multirow{2}{*}{34060} & \multirow{2}{*}{0.0001} \\
\hline & $B$ & 205 & 0.89 & 0.32 & 0.81 & & \\
\hline
\end{tabular}

El rendimiento promedio en la producción de tablas escuadradas estimado en los aserraderos evaluados fue de $27.74 \%$ en el aserradero A y de $48.84 \%$ para el aserradero B, cuyos resultados son: por un lado, inferior con el $7.26 \%$ y, por el otro, superior con el 3.84 \% en relación a los valores promedio de $35 \%$ y $45 \%$ determinados con similares tipos de aserrío de acuerdo al estudio realizado en la especie Pinus radiata por (Aldás, 2014). Al comparar el rendimiento del aserradero B, su valor es ligeramente inferior con el $1.38 \%$ en referencia al $50.22 \%$ reportado para Eucalyptus spp al realizar el proceso de transformación primaria con sierra de cinta vertical en un aserradero de Cartago, Costa Rica (Ureña, 2017). Por su parte, De Souza et al. (2007) en su trabajo con Eucalyptus spp en el estado de Minas Gerais, Brasil, determinó rendimientos de 38 \% con variaciones del 33 a $61 \%$. Lo anterior significa que por cada metro cúbico de madera en rollo $\left(\mathrm{m}^{3} \cdot \mathrm{r}\right)$ procesada, se obtuvo $117 \mathrm{pt}\left(0.277 \mathrm{~m}^{3}\right)$ y $207 \mathrm{pt}\left(0.488 \mathrm{~m}^{3}\right)$ de madera aserrada, respectivamente. Al proyectar a $1.000 \mathrm{pt}\left(2.36 \mathrm{~m}^{3}\right)$ de madera aserrada se requieren entre $8 \mathrm{~m}^{3}$ de madera en rollo para su aserrío en sierra circular y de $5.49 \mathrm{~m}^{3}$ en el sistema sierra circular y de cinta.

En el aserradero A, la productividad varío desde 0.29 a $2.57 \mathrm{~m}^{3}$ de madera en rollo por hora efectiva de trabajo con un promedio de $1.17 \mathrm{~m}^{3} \mathrm{r} \cdot \mathrm{h}^{-1}$, equivalentes a aserrar $532 \mathrm{pt} \cdot \mathrm{h}^{-1}$, que distribuidos entre el número de operarios que participan en el proceso, en este caso un promedio de cinco, resulta entonces en una eficiencia de 106 pies tablares/hombre-máquina/hora. En cambio, en el aserradero B, se determinó valores de 0.20 a $2.59 \mathrm{~m}^{3}$ de madera en rollo por hora, con un valor promedio de $0.89 \mathrm{~m}^{3} \cdot \cdot \mathrm{h}^{-1}$, valor que corresponde a elaborar $358 \mathrm{pt} \cdot \mathrm{h}^{-1}$, con cinco operarios trabajando presenta una eficiencia de 72 pies tablares/hombre-máquina/hora en el sistema conformado por una sierra circular y de cinta. La productividad del aserradero B resultó inferior con el 25.21 \% en relación al obtenido por Ureña (2017), quién determinó mediante el estudio de 39 trozas de Eucalyptus spp con diámetros entre 20 a $55 \mathrm{~cm}$ un rendimiento de $1.19 \mathrm{~m}^{3} \mathrm{r} \cdot \mathrm{h}^{-1}$

\subsection{Tiempos y rendimientos por categoria diamétrica y conicidad de las trozas}

\section{Categoría diamétrica del aserradero A}

El tiempo total estimado para aserrar 1000 pt (min.) tomó valores desde 85.12, 104.5 y 161.38 min. correspondientes a las categorías diamétricas: 14-25, 25,1-35 y 35,1-45 cm, respectivamente (tabla 8). 
Tabla 8. Tiempos y rendimientos por categoría diamétrica en el proceso con sierra circular aserradero A

\begin{tabular}{|c|c|c|c|}
\hline \multirow{2}{*}{ Variable } & \multicolumn{3}{|c|}{ Categoría diamétrica $(\mathrm{cm})$} \\
\hline & $14-25$ & 25.1-35 & $35.1-45$ \\
\hline \multicolumn{4}{|l|}{ Características de las trozas aserradas } \\
\hline Diámetro mayor con corteza $(\mathrm{m})$ & $0.23(0.03)$ & $0.31(0.02)$ & $0.41(0.02)$ \\
\hline Diámetro menor con corteza $(\mathrm{m})$ & $0.21(0.02)$ & $0.29(0.02)$ & $0.39(0.02)$ \\
\hline Longitud de la troza (m) & $2.52(0.03)$ & $2.52(0.03)$ & $2.52(0.03)$ \\
\hline Volumen total con corteza $\left(\mathrm{m}^{3} \cdot r\right)$ & 7.19 & 14.25 & 17.58 \\
\hline \multicolumn{4}{|c|}{ Productos obtenidos del aserrío } \\
\hline Total de tablas generadas ( $\mathrm{n}$ ) & 201 & 416 & 637 \\
\hline Tablas promedio por troza (n) & $3(1)$ & $5(1)$ & $11(2)$ \\
\hline Volumen total de las tablas aserradas $\left(\mathrm{m}^{3}\right)$ & 1.83 & 3.8 & 5.87 \\
\hline \multicolumn{4}{|c|}{ Tiempo para aserrar 1000 pt (min.) en la sierra circular } \\
\hline Tiempo de descargue de trozas en aserradero & $12.7(5.91)$ & $12.19(5.68)$ & $17.34(8.06)$ \\
\hline Tiempo de traslado de troza a la sierra circular & $2.35(0.05)$ & $2.42(0.08)$ & $2.89(0.6)$ \\
\hline Tiempo de carga & $2.76(0.17)$ & $2.62(0.18)$ & $3.67(0.87)$ \\
\hline Tiempo de avance & $33.48(5.44)$ & $46.38(12.79)$ & $75.59(6.8)$ \\
\hline Tiempo de retrocesos & $10.17(6.23)$ & $16.14(8.94)$ & $21.52(3.36)$ \\
\hline Tiempo de volteos de las trozas & $9.7(3.67)$ & $11.14(4.08)$ & $26.72(5.56)$ \\
\hline Tiempo de traslado y apilado de tablas & $1.64(0.36)$ & $1.71(0.39)$ & $1.68(0.35)$ \\
\hline Tiempo justificado & $10.04(6.18)$ & $9.64(5.91)$ & $9.99(7.67)$ \\
\hline Tiempo no justificado & $2.26(0.91)$ & $2.29(1.01)$ & $1.97(0.75)$ \\
\hline Total proceso sierra circular & 85.1 & 104.53 & 161.37 \\
\hline \multicolumn{4}{|c|}{ Indicadores de productividad en la operación de aserrío } \\
\hline Tiempo total de aserrado para 1000 pt (min.) & $85.12(9.93)$ & $104.5(19.68)$ & $161.38(14.33)$ \\
\hline Rendimiento con corteza (\%) & $24.57(7.3)$ & $26.88(4.29)$ & $33.18(3.51)$ \\
\hline Productividad $\left(\mathrm{m}^{3} \cdot \mathrm{h}^{-1}\right)$ & $0.73(0.29)$ & $1.21(0.23)$ & $1.69(0.34)$ \\
\hline
\end{tabular}

Con los resultados obtenidos, se distingue un aumento del tiempo de aserrado a medida que se incrementa el volumen de madera procesada, como ocurrió con el tiempo de la tercera categoría diamétrica, el cual se amplía en 1.54 veces en relación con el tiempo (104.5 min.) de la segunda clase diamétrica al trabajar con sierra circular (tabla 8). La tendencia del rendimiento con corteza muestra un incremento desde $24.57,26.88$ y $33.18 \%$ a medida que aumenta el diámetro de las trozas de forma general en el aserradero A. Siendo el diámetro de la troza uno de los factores de incidencia en el proceso de aserrío (Álvarez et al., 2003). En referencia a la variable productividad $\left(\mathrm{m}^{3} \cdot \mathrm{h}^{-1}\right)$ al aserrar con sierra circular, esta se incrementa a medida que aumenta la clase diamétrica, con valores desde $0.73 \mathrm{~m}^{3} \cdot \mathrm{h}^{-1}$ en la categoría de 14-25 hasta 1.69 $\mathrm{m}^{3} \cdot \mathrm{h}^{-1}$ para el rango entre $35.1-45 \mathrm{~cm}$ (Tabla 8), confirman lo determinado por Biasi y Rocha (2003) quienes evaluaron el rendimiento de madera aserrada de Pinus elliottii en base a cuatro clases de diámetro (8-18 cm, $14-24 \mathrm{~cm}, 20-32 \mathrm{~cm}$ y $33-45 \mathrm{~cm})$, determinaron un incremento de productividad con el aumento de las clases diamétricas.

\section{Categoría diamétrica del aserradero B}

Los tiempos totales proyectados para aserrar 1000 pt (min.), en el aserradero B son de 146.88, 189.4, 240.49 y 315,35 minutos correspondientes a las categorías diamétricas: 14-25, 25.1-35, 35.1- 45 y $45.1-55 \mathrm{~cm}$ respectivamente. 
Tabla 9. Tiempos y rendimientos por categoría diamétrica con sierra circular + sierra de cinta del aserradero B

\begin{tabular}{|c|c|c|c|c|}
\hline \multirow{2}{*}{ Variable } & \multicolumn{4}{|c|}{ Categoría diamétrica $(\mathrm{cm})$} \\
\hline & $14-25$ & 25.1-35 & $35.1-45$ & $45.1-55$ \\
\hline Diámetro mayor con corteza (m) & $0.23(0.03)$ & $0.31(0.03)$ & $0.43(0.03)$ & $0.5(0.01)$ \\
\hline Diámetro menor con corteza (m) & $0.21(0.03)$ & $0.28(0.2)$ & $0.38(0.03)$ & $0.47(0.01)$ \\
\hline Longitud de la troza (m) & $2.44(0.14)$ & $2.48(0.09)$ & $2.49(0.02)$ & $2.51(0.01)$ \\
\hline Volumen total con corteza $\left(\mathrm{m}^{3} \cdot r\right)$ & 11.85 & 10.61 & 4.42 & 0.92 \\
\hline \multicolumn{5}{|c|}{ Productos obtenidos del aserrío } \\
\hline Total de tablas generadas ( $n$ ) & 632 & 383 & 102 & 13 \\
\hline Tablas promedio por troza (n) & $5(1)$ & $6(1)$ & $7(1)$ & $7(2)$ \\
\hline Volumen total de las tablas aserradas $\left(\mathrm{m}^{3}\right)$ & 6.44 & 4.29 & 1.11 & 0.13 \\
\hline \multicolumn{5}{|c|}{ Tiempo para aserrar $1000 \mathrm{pt}$ (min.) en la sierra circular } \\
\hline Tiempo de descargue de trozas en aserradero & $11.20(3.71)$ & $11.86(3.78)$ & $12.65(4.22)$ & $9.92(5.9)$ \\
\hline Tiempo de traslado de troza a la sierra circular & $3.02(2.43)$ & $4.33(2.87)$ & $3.80(1.4)$ & $18.05(7.83)$ \\
\hline Tiempo de carga & $3.58(2.89)$ & $5.25(4.54)$ & $6.32(4.96)$ & $10.26(2.77)$ \\
\hline Tiempo de avance & $17.64(7.24)$ & $24.97(10.81)$ & $25.77(10.37)$ & $50.54(17.459$ \\
\hline Tiempo de retrocesos & $11.88(6.67)$ & $13.99(10.86)$ & $7.48(3.07)$ & $11.95(4.93)$ \\
\hline Tiempo de volteos de las trozas & $13.79(44.67)$ & $11.86(7.33)$ & $11.27(8.28)$ & $38.2(11.77)$ \\
\hline Tiempo de traslado y apilado de bloques & $1.83(0.19)$ & $2.02(0.32)$ & $2.27(0.34)$ & $2.36(0.00)$ \\
\hline Tiempo justificado & $15.19(5.80)$ & $15.84(7.39)$ & $16.66(5.29)$ & $13.89(4.18)$ \\
\hline Tiempo no justificado & $4.3(0.95)$ & $4.46(0.99)$ & $4.55(0.65)$ & $4.33(1.29)$ \\
\hline \multicolumn{5}{|c|}{ Tiempo para aserrar 1000 pt (min.) en la sierra de cinta } \\
\hline Tiempo de carga a sierra de cinta & $1.91(0.57)$ & $3.11(1.41)$ & $5.19(0.42)$ & $5.9(0.05)$ \\
\hline Tiempo de avance & $24.6(4.80)$ & $35.77(13.26)$ & $56.23(3.08)$ & $58.51(3.8)$ \\
\hline Tiempo de retroceso & $22.73(5.17)$ & $35.03(13.84)$ & $55.91(4.41)$ & $56.7(1.5)$ \\
\hline $\begin{array}{l}\text { Tiempo de volteos para colocar bloques en la par- } \\
\text { te superior de la sierra de cinta }\end{array}$ & $10.89(2.92)$ & $16.35(6.90)$ & $27.24(0.72)$ & $29.18(2.45)$ \\
\hline $\begin{array}{l}\text { Tiempo de traslado y apilado de tablas que ingre- } \\
\text { saron a la sierra de cinta }\end{array}$ & $1.44(0.22)$ & $1.70(0.20)$ & $2.26(0.51)$ & $2.63(0.1)$ \\
\hline Tiempo justificado en la sierra de cinta & $1.54(0.03)$ & $1.55(0.03)$ & $1.54(0.03)$ & $1.56(0.01)$ \\
\hline Tiempo no justificado en la sierra de cinta & $1.32(0.03)$ & $1.31(0.05)$ & $1.32(0.04)$ & $1.38(0.1)$ \\
\hline Tiempo total en la sierra circular y de cinta & 146.86 & 189.4 & 240.46 & 315.36 \\
\hline \multicolumn{5}{|c|}{ Indicadores de productividad en la operación de aserrío } \\
\hline Tiempo total de aserrado para 1000 pt (min.) & $146.88(49.60)$ & $189.4(45.05)$ & $240.49(20.05)$ & $315.35(35.61)$ \\
\hline Rendimiento con corteza (\%) & $55.47(13.35)$ & $41.22(13.19)$ & $25.34(6.54)$ & $14.45(6.13)$ \\
\hline Productividad $\left(\mathrm{m}^{3} \cdot \mathrm{h}^{-1}\right)$ & $0.86(0.29)$ & $0.96(0.38)$ & $0.81(0.23)$ & $0.52(0.26)$ \\
\hline
\end{tabular}

Se asume, de manera general, que a medida que aumenta el volumen procesado se requiere de más tiempo para su aserrado. El comportamiento del rendimiento con corteza muestra una disminución cuando aumenta el diámetro de las trozas cuyos valores se encuentran desde $55.47 \%(14-25 \mathrm{~cm})$ hasta $14.45 \%$ (45.1-55 cm), resultados que obedecen a un diferente número de trozas agrupadas fundamentalmente para las clases diamétricas de $35.1-45 \mathrm{~cm}$ y 45.1-55 cm respectivamente (Tabla 9). 
Las trozas al transformarse a madera aserrada sin aplicar ningún tipo de clasificación ni definición adecuada de un modelo de corte para cada clase diamétrica, tal condición, causa la disminución de su nivel aprovechamiento, lo cual genera una mayor cantidad de subproductos del proceso (Rocha, 2002). En cambio, la variable productividad al aserrar con el sistema sierra circular y de cinta, muestra un comportamiento irregular que se incrementa en la segunda clase diamétrica $(25.1-35 \mathrm{~cm})$ con $0.10 \mathrm{~m}^{3} \cdot \mathrm{h}^{-1}$ en referencia al valor 0.86 que corresponde a la primera categoría diamétrica, luego disminuye a 0.81 y $0.52 \mathrm{~m}^{3} \mathrm{~h}^{-1}$, valores pertenecientes a la tercera y cuarta clase diamétrica, las cuales tuvieron un menor número de trozas para su evaluación en el aserradero B. El menor tiempo total de aserrado para 1000 pt (min.), se registró en la clase diamétrica de 14-25 cm del aserradero A provisto de sierra circular con el valor promedio de 85.12 min., y el mayor tiempo total de aserrado se determinó para la clase diamétrica de $46-55 \mathrm{~cm}$ en el aserradero B, cuyo proceso de operación utiliza el sistema sierra circular y de cinta con el índice de 315.35 min. (Tabla 9).

\section{Conicidad de trozas del aserradero $A$}

Con sierra circular el tiempo total de aserrado para 1000 pt, tiene un comportamiento similar al presentar valores estimados de 114.35 minutos, 110.75 y 109.44 minutos pertenecientes a las categorías de conicidad de 0-1, 1.1-2 y 2.1-3 cm (Tabla 10). Asimismo, las variables rendimiento y productividad mostraron similitud con valores de $27.12 \%, 28.3 \%, 31.48 \%$ y de $1.15,1.19$ y de $1.27 \mathrm{~m}^{3} \cdot \mathrm{h}^{-1}$ para las tres clases agrupadas como parte de la investigación (Tabla 10).

Tabla 10. Tiempos y rendimientos por conicidad de trozas en el aserradero A

\begin{tabular}{|c|c|c|c|}
\hline \multirow{2}{*}{ Variable } & \multicolumn{3}{|c|}{ Categorías de conicidad de las trozas (cm) } \\
\hline & $0-1$ & $1.1-2$ & $2.1-3$ \\
\hline \multicolumn{4}{|c|}{ Características de las trozas aserradas } \\
\hline Diámetro mayor con corteza (m) & $0,31(0.07)$ & $0.31(0.07)$ & $0.32(0.07)$ \\
\hline Diámetro menor con corteza $(\mathrm{m})$ & $0.29(0.07)$ & $0.28(0.07)$ & $0.26(0.07)$ \\
\hline Longitud de la troza (m) & $2.52(0.02)$ & $2.52(0.03)$ & $2.52(0.02)$ \\
\hline Volumen total con corteza $\left(\mathrm{m}^{3} \cdot r\right)$ & 22.51 & 15.3 & 1.22 \\
\hline \multicolumn{4}{|l|}{ Productos obtenidos del aserrío } \\
\hline Total de tablas generadas ( $\mathrm{n}$ ) & 706 & 503 & 45 \\
\hline Volumen total de las tablas aserradas $\left(\mathrm{m}^{3}\right)$ & 6.48 & 4.62 & 0.4 \\
\hline \multicolumn{4}{|c|}{ Tiempo para aserrar 1000 pt (min.) en la sierra circular } \\
\hline Tiempo de descargue de trozas en aserradero & $14.34(7.42)$ & $12.99(6.04)$ & $12.72(3.24)$ \\
\hline Tiempo de traslado de troza a la sierra circular & $2.98(0.46)$ & $2.50(0.26)$ & $2.51(0.35)$ \\
\hline Tiempo de carga & $2.96(0.73)$ & $2.92(0.5)$ & $2.82(0.59)$ \\
\hline Tiempo de avance & $49.56(18.83)$ & $48.95(19.65)$ & $49.46(16.61)$ \\
\hline Tiempo de retrocesos & $15.49(8.51)$ & $15.57(7.65)$ & $13.21(8.93)$ \\
\hline Tiempo de volteos de las trozas & $15.00(8.66)$ & $14.3(8.19)$ & $16.8(9.02)$ \\
\hline Tiempo de traslado y apilado de tablas & $1.68(0.4)$ & $1.68(0.34)$ & $1.54(0.21)$ \\
\hline Tiempo justificado & $10.12(7.37)$ & $9.67(5.17)$ & $8.20(4.66)$ \\
\hline Tiempo no justificado & $2.20(0.89)$ & $2.19(0.98)$ & $2.18(0.61)$ \\
\hline Total proceso sierra circular & 114.33 & 110.77 & 109.44 \\
\hline \multicolumn{4}{|c|}{ Indicadores de productividad en la operación de aserrío } \\
\hline Tiempo total de aserrado para 1000 pt (min.) & 114.35 (35.95) & $110.75(32.19)$ & $109.44(27.81)$ \\
\hline Rendimiento con corteza (\%) & $27.12(6.2)$ & $28.3(6.52)$ & $31.48(6.93)$ \\
\hline Productividad $\left(\mathrm{m}^{3} \cdot \mathrm{h}^{-1}\right)$ & $1.15(0.45)$ & $1.19(0.49)$ & $1.27(0.53)$ \\
\hline
\end{tabular}




\section{Conicidad de trozas del aserradero $B$}

El tiempo total de aserrado para 1000 pt en el aserradero B, se determinó que aumenta cuando se incrementa el grado de conicidad variando desde 154.06 minutos $(0-1 \mathrm{~cm} / \mathrm{m})$ hasta 282.62 minutos, para la categoría diamétrica de 5-6 $(\mathrm{cm} / \mathrm{m})$. Mientras que el rendimiento presentó un comportamiento atípico al mostrar valores desde $52.52 \%$ perteneciente a la primera categoría de conicidad $(0-1 \mathrm{~cm} / \mathrm{m})$, para la segunda clase de conicidad $(1.1-2 \mathrm{~cm} / \mathrm{m})$ su valor disminuye al $45.62 \%$, luego se incrementa al $48.82 \%(2.1-3 \mathrm{~cm} / \mathrm{m})$ y se reduce en la sexta clase de conicidad $(5.1-6 \mathrm{~cm} / \mathrm{m})$ con el $28.28 \%$ (Tabla 11).

Tabla 11. Tiempos y rendimientos por conicidad de trozas en el aserradero B

\begin{tabular}{|c|c|c|c|c|c|c|}
\hline \multirow{2}{*}{ Variable } & \multicolumn{6}{|c|}{ Categorías de conicidad de las trozas $(\mathrm{cm})$} \\
\hline & $0-1$ & $1.1-2$ & 2.1-3 & $3.1-4$ & 4.1-5 & $5.1-6$ \\
\hline \multicolumn{7}{|l|}{ Características de las trozas aserradas } \\
\hline Diámetro menor con corteza (m) & $0.23(0.05)$ & $0.25(0.07)$ & $0.27(0.09)$ & $0.3(0.08)$ & $0.31(0.07)$ & $0.27(0.00)$ \\
\hline Longitud de la troza (m) & $2.47(0.11)$ & $2.49(0.14)$ & $2.49(0.11)$ & $2.48(0.03)$ & $2.32(0.24)$ & $2.48(0.00)$ \\
\hline \multicolumn{7}{|c|}{ Productos obtenidos del aserrío } \\
\hline Total de tablas generadas (n) & 536 & 482 & 65 & 25 & 15 & 7 \\
\hline Volumen total de las tablas aserradas $\left(\mathrm{m}^{3}\right)$ & 5.67 & 5.07 & 0.66 & 0.35 & 0.14 & 0.07 \\
\hline \multicolumn{7}{|c|}{ Tiempo para aserrar en la sierra circular 1000 pt (min.) } \\
\hline Tiempo de carga & $4.42(3.58)$ & $4(3.86)$ & $5.53(3.27)$ & $6.13(7.15)$ & $3.93(2.59)$ & $2.12(0.00)$ \\
\hline Tiempo de avance & $20.25(9.58)$ & $20.41(9.69)$ & $22.61(11.57)$ & $22.14(10.41)$ & $25.89(8.77)$ & $48.53(0.00)$ \\
\hline Tiempo de retrocesos & $13.25(7.31)$ & $11.25(8.85)$ & $10.03(6.16)$ & $10.66(12.36)$ & $15.01(13.85)$ & $6.29(0.00)$ \\
\hline Tiempo de volteos de las trozas & $11.3(6.6)$ & $16.35(55.06)$ & $9.97(6.54)$ & $7.5(4.49)$ & $10.83(6.28)$ & $20.14(0.0)$ \\
\hline Tiempo de traslado y apilado de tablas & $1.89(0.23)$ & $1.92(0.28)$ & $1.99(0.51)$ & $2.23(0.39)$ & $2.12(0.33)$ & $1.89(0.0)$ \\
\hline Tiempo justificado & $15.51(6.41)$ & $15.49(6.32)$ & $16.21(4.34)$ & $14.11(6.19)$ & $9.21(2.54)$ & $20.22(0.0)$ \\
\hline Tiempo no justificado & $4.28(0.97)$ & $4.4(0.94)$ & $4.41(0.47)$ & $4.97(1.17)$ & $4.97(0.39)$ & $6.21(0.0)$ \\
\hline Subtotal proceso en sierra circular & 85.4 & 89.21 & 87.16 & 85.73 & 89.76 & 125.75 \\
\hline $\begin{array}{l}\text { Tiempo de traslado y apilado de tablas } \\
\text { que ingresaron a la sierra de cinta }\end{array}$ & $1.51(0.26)$ & $1.6(0.34)$ & $1.74(0.49)$ & $2.15(0.41)$ & $2.26(0.62)$ & $2.70(0.00)$ \\
\hline Tiempo justificado en la sierra de cinta & $1.54(0.03)$ & $1.55(0.04)$ & $1.55(0.04)$ & $1.53(0.02)$ & $1.55(0.02)$ & $1.58(0.00)$ \\
\hline Tiempo no justificado en la sierra de cinta & $1.32(0.05)$ & $1.32(0.03)$ & $1.31(0.01)$ & $1.33(0.06)$ & $1.33(0.00)$ & $1.39(0.00)$ \\
\hline Subtotal proceso en sierra de cinta & 68.66 & 87.29 & 98.43 & 122.44 & 144.18 & 156.89 \\
\hline Total procesos sierra circular y de cinta & 154.06 & 176.5 & 185.59 & 208.17 & 233.94 & 282.64 \\
\hline Tiempo total de aserrado para 1000 pt (min.) & $154.06(33.51)$ & 176.5 (71.99) & $185.58(51.52)$ & $208.17(61.18)$ & $233.94(25)$ & $282.62(0.00)$ \\
\hline Rendimiento con corteza (\%) & $52.52(12.64)$ & $45.62(16.7)$ & 48.82 (31.91) & 37.08 (3.31) & $29.97(7.7)$ & $28.28(0.00)$ \\
\hline Productividad $\left(\mathrm{m}^{3} \cdot \mathrm{h}^{-1}\right)$ & $0.89(0.28)$ & $0.88(0.38)$ & $0.91(0.27)$ & $0.99(0.27)$ & $0.75(0.23)$ & $0.58(0.00)$ \\
\hline
\end{tabular}


Resultados obtenidos debido a la incidencia promovida por el mayor número de trozas agrupadas en la primera categoría de conicidad con un número de 103 en contraste con la categoría de 5.1-6 donde se contabilizó una sola troza (Tabla 11).

La variable productividad presentó un comportamiento semejante entre la primera y segunda categoría de conicidad con valores de 0.89 y $0.88 \mathrm{~m}^{3} \cdot \mathrm{h}^{-1}$, posteriormente se incrementa para la categoría de $3.1-4$ con $0.99 \mathrm{~m}^{3} \cdot \mathrm{h}^{-1}$ hasta disminuir a un valor de $0.58 \mathrm{~m}^{3} \cdot \mathrm{h}^{-1}$ correspondiente a la clase de $5.1-6 \mathrm{~cm} / \mathrm{m}$. Resultados que coinciden en relación a lo expuesto por Manhiça et al. (2012). quienes indican que la conicidad incide sobre las primeras piezas aserradas después de retirar las costeras siempre presentan una forma irregular. y que al cuadrarlas generalmente se reducen en anchos y largos. propiciando la obtención de rendimientos relativamente bajos en ciertas clases diamétricas o en el rendimiento general del aserrado.

El efecto de la conicidad se cumple de forma parcial, según lo expresado por Liu y Zhang (2005) y Álvarez et al. (2010), quienes puntualizan que la conicidad de la troza es una de las variables que inciden en los rendimientos de madera aserrada.

\subsection{Prueba de Mann Whitney}

Al aplicar la Prueba de Mann Whitney se distingue dos diferencias significativas para el diámetro mayor y menor de las trozas entre los dos aserraderos $(p<0.0001)$. En cambio, la conicidad no presentó efectos significativos ( $p=0.436$; tabla 12) sobre el rendimiento en madera aserrada eucalipto

Tabla 12. Prueba de Mann Whitney para el diámetro mayor, menor y la conicidad de las trozas

\begin{tabular}{|c|c|c|c|c|c|c|c|}
\hline Variable & Aserradero & $\mathrm{n}$ & Media & Desv Std & Mediana & W & $P$ \\
\hline \multirow{2}{*}{ D. Max.m } & A & 210 & 0.31 & 0.07 & 0.3 & \multirow{2}{*}{35734} & \multirow{2}{*}{0.0001} \\
\hline & $B$ & 205 & 0.27 & 0.07 & 0.26 & & \\
\hline \multirow{2}{*}{ D. Mín. m } & A & 210 & 0.28 & 0.07 & 0.28 & \multirow{2}{*}{35483} & \multirow{2}{*}{0.0001} \\
\hline & B & 205 & 0.24 & 0.06 & 0.23 & & \\
\hline \multirow{2}{*}{ Conicidad } & $A$ & 210 & 1.00 & 0.76 & 0.93 & \multirow{2}{*}{35483} & \multirow{2}{*}{0,436} \\
\hline & $B$ & 205 & 1.12 & 0.93 & 1.00 & & \\
\hline
\end{tabular}

\section{Conclusiones y recomendaciones}

Los tiempos estimados para procesar 1.000 pt $\left(2.36 \mathrm{~m}^{3}\right)$ es menor con el $32.64 \%$ al trabajar con sierra circular del aserradero $A$, en referencia a los 167.36 minutos del aserradero $B$, con 46.16 minutos para la elaboración de bloques en la sierra circular, y de 73.07 minutos en la fase complementaria de bloques a tablas con la sierra de cinta. En cambio, en el rendimiento resultó superior en el aserradero B, con el $21.1 \%$ en relación $27.74 \%$ del aserradero A, por cada metro cúbico de madera en rollo $\left(\mathrm{m}^{3} \cdot \mathrm{r}\right)$ procesada, se obtuvo 207 pt $\left(0.488 \mathrm{~m}^{3}\right)$ y 117 pt $\left(0.277 \mathrm{~m}^{3}\right)$ de madera aserrada respectivamente.

En el aserradero $\mathrm{A}$, a medida que aumenta el diámetro de las trozas existe un incremento en el rendimiento y productividad, en cambio en el aserradero B, no evidencia influencia del diámetro en el rendimiento de la madera aserrada, debido al diferente número de trozas valoradas por clase diamétrica en cada fábrica. 
El efecto del diámetro mayor y menor fue significativo para las clases diamétricas evaluadas, pero la conicidad no fue significativa al momento de elaborar tablas de eucalipto utilizando los sistemas sierra circular en el aserradero A y el sistema combinado sierra circular más sierra de cinta del aserradero B.

Desarrollar estudios complementarios de tiempos y rendimientos que implica la conversión de madera en rollo a madera aserrada de eucalipto y otras especies a nivel de aserradero considerando la agrupación de trozas por clases diamétricas, longitud de trozas, su calidad, conicidad, los sistemas combinados de sierra circular más la de cinta, y otros factores tendientes a reducir costos de producción y la mejora del proceso de aserrío para otros aserraderos con similares instalaciones o procesos.

\section{Bibliografía}

Aldás, G. (2014). Rendimiento en el proceso de transformación de madera rolliza a madera escuadrada de pino (Pinus radiata D.Don), con dos tipos de aserradero, en la ciudad de Riobamba. Tesis de pregrado. Facultad de Recursos Naturales. Escuela Superior Politécnica de Chimborazo, Ecuador.

Álvarez, D., Andrade, F., Chávez, P., Estévez, I., y García, J. M. (2003). Análisis matemático para elevar la eficiencia de los aserraderos con sierras de banda. Revista Chapingo Serie Ciencias Forestales y del Ambiente, 9(1):89-94.

Álvarez, D., Estévez, I., Domínguez, A., García, O., Alaejos, J., y Rodríguez, J. C. (2010). Improvement of the lumber recovery factory with low environmental impact in Pinar del Río, Cuba. The International Forestry Review 12(5): 303.

Balzarini, M. G., Gonzalez, L., Tablada, M., Casanoves, F., Di Rienzo, J. A., y Robledo, C. W. (2008). Infostat. Manual del Usuario. Córdova, Argentina: Brujas.

Biasi, C. P.; Rocha, M. P. (2003). Rendimento em madeira serrada de Pinus elliottii. Congresso Florestal Brasileiro, 8, 2003, São Paulo. Anais. São Paulo: [s.n.].

Biasi, C. P., y Pereira Da Rocha, M. (2007). Rendimento em madeira serrada e quantificação de resíduos para três espécies tropicais. Floresta, 37(1): 95-108.

Calvache, J. (2016). La investigación científica como alternativa en la formación profesional. Bogotá, Colombia: CEPUN.

García, J. D., Morales, L., y Valencia, S. 2001. Coeficientes de aserrío para cuatro aserraderos banda en el Sur de Jalisco. Foresta-AN. Nota técnica Núm. 5. UAAAN, Saltillo, Coah. 12 p.

Guallpa, M., Rosero, S., Samaniego, M., y Cevallos, E. (2016). Caracterización edáfica y dasométrica de una plantación de Eucalyptus globulus Labill y propuesta de manejo en la zona estepa espinosa Montano Bajo, Riobamba, Ecuador. Enfoque UTE, 7 (3), 26-40.

Guallpa, M., Rosero, S., Montenegro, G., y Quinchuela, D (2018). Estimación de los residuos forestales en los aserraderos de tres cantones, Zona 3 interandina, Ecuador. European Scientific Journal, 14 (30), 228-239.

Guallpa, M., Lara, N., Espinoza, M., Guilcapi, E., y Fosado, O. (2019). Valoración cualitativa de una plantación de Eucalyptus globulus Labill en el sector de Licto, Riobamba, Ecuador. Polo del Conocimiento, 4 (4), 126-152.

Ecuador Forestal. (Mayo de 2010). Organización Ecuador Forestal. http://ecuadorforestal.org/wp-content/uploads/2010/05/Instructivo- Cubicaci\%C3\%B3n-de-Madera.pdf

Ecuador Forestal. (Julio de 2013). Organización Ecuador Forestal. http://ecuadorforestal.org/wp-content/ uploads/2013/03/PE_Industrias.pdf

INAB, ITTO, 2016. Guía para estudios de rendimiento de transformación primaria. Guatemala, SERIE TÉCNICA GT-011 (2016) 47 paginas. 
López, N., y Muñoz, J. (2017). La producción forestal una actividad con alto potencial en el Ecuador requiere un cambio de visión. Bosques Latitud Cero, 7 (1), 69-76.

Liu, C. y Zhang, S. Y. (2005). Models for predicting product recovery using selected tree characteristics of black spruce. Canadian Journal Forest Resources 35: 930-937.

Manhiça, A. A., Pereira Da Rocha, M., y Timofeiczyk-Júnior, R. (2012). Rendimento no desdobro de Pinus sp. utilizando modelos de corte numa serraria de pequeño porte. Floresta, 42 (2): 409-420.

Ministerio del Ambiente del Ecuador, 2011. Descripción de las Cadenas Productivas de Madera en el Ecuador. Quito. 95p, Quito: MAE-ITTO.

Murara Junior, M., Rocha, M. P., y Timofeiczyk Junior, R. (2005). Rendimiento em madeira serrada de Pinus taeda para duas metodologias de desdobro. Floresta 35 (3): 473- 483.

Nájera, J., Aguirre, O., Treviño, E., Jiménez, J., Jurado, E., y Corral, J. (2011). Rendimiento volumétrico y calidad dimensional de la madera aserrada en aserraderos de El Salto, Durango. Revista Mexicana de Ciencias Forestales, 2(4): 75-89.

Nájera, J., Adame, G., Méndez, J., Vargas, B., Cruz, F., Hernández, F., y otros. (2012). Rendimiento de la madera aserrada en dos aserraderos privados de El Salto, Durango, México. Investigación y Ciencia de la Universidad Autónoma de Aguascalientes México, 20 (55): 11-23.

PRO ECUADOR. (2013). Muebles y productos de madera - Pro Ecuador. Obtenido de http://www.proecuador.gob.ec/wp-content/uploads/2015/06/Perfiles-de-Inversiones-Promocion-de-Inversiones/ Perfiles-de-Inversion/Muebles-y-Productos-de-Madera.pdf

Rocha, M. (2002). Técnicas e planejamento de serrarías. Serie didáctica 02/01. FUPEP, Curitiba. 121p.

Romahn de la V. C. F., Ramírez, M., y Treviño, J. L. (1987). Dendrometría. Serie de apoyo académico Núm. 26. División de Ciencias Forestales., Texcoco, México: Universidad Autónoma Chapingo.

Rondeux, J. (2010). Medición de árboles y masas forestales. España: Mundi- Prensa.

Rivas, D. (2013). "Guía de cubicación de madera". Consultado el 10 de marzo de 2018. Disponible en http:// www.rivasdaniel.com/pdf/GUIA_DE_CUBICACION_MADERA.pdf

Scanavaca, L., y García, J. (2003). Rendimento em madeira serrada de Eucalyptus urophylla. Scientia Forestalis, 63: 32-43.

Valério, A., Farinha, W., Tavolca, C., y H, S. (2007). Cuantificação de resíduos e rendimento no desdobro de Araucaria angustifolia (Bertol) 0. Kuntze. Floresta, 37 (3): 387-398.

Valério, Á. F., Watzlawick, L. F., Balbinot, R.; Wincker, M. V., y Filho, A. F. (2009). Modelagem para a estimativa do rendimento no desdobro de toras de Araucaria angustifolia (Bertol) Kuntze. Floresta, 39(3): 619-628

Vignote, P., y Martínez, R. (2005). Tecnología de la madera. Madrid, España: Ediciones Mundi-Prensa Libros.

Villagómez, L., y García, A. (1986). El estudio de trabajo y su aplicación en las operaciones de abastecimiento forestal. Ciencia Forestal en México, 59(11): 162-180.

Ureña, K. (2017). Análisis del rendimiento y eficiencia actual en la producción de madera aserrada, en el aserradero El Almendro, Cartago, Costa Rica. Tesis de pregrado. Instituto Tecnológico de Costa Rica.

Vital, B. R. (2008). Planejamento e operação de serrarias. Viçosa, MG, Editora UFV. 